\title{
Design of a Pneumatic Soft Finger with Two Joints
}

\author{
Chengyu LI, Dengyu XIE, Hongjun LI ${ }^{1}$ \\ Affiliation Faculty of Mechanical Engineering and Automation, Zhejiang Sci-Tech \\ University, Xiasha Higher Education Zone, Hangzhou 310018, China
}

\begin{abstract}
In this paper, a pneumatic soft finger with two joints is designed. The finger includes multiple air chambers, and the endoskeleton is embedded in the bottom layer of the pneumatic soft finger. The magnitude of air pressure controls the finger bending angle/moment. The endoskeleton and mold are fabricated by $3 \mathrm{D}$ printing technology with PLA material, while the finger mainbody is made of silica gel. The finger bending performance is analyzed by both numerical modelling and experiments. The finite element method is used to build the finger model. The silica gel material properties are obtained by the uniaxial tensile test and the Yeoh constitutive model is selected for the stress and strain relationships. It was found that the finger was able to bend under applied internal pressure. The numerical model successfully predicted the finger deformation and stress distribution. The curve of air pressure against bending angle was obtained.
\end{abstract}

Keywords. Pneumatic soft fingers, endoskeleton, 3D printing, silica gel

\section{Introduction}

In the 1960s, a company in the United States successfully developed the world's first industrial robot Unimate, which greatly promoted the progress of robotics and the development of world industrial technology, which marked that industrial technology had entered a new era of robots [1-3]. With the development of robot technology, the application scope of robots has gradually expanded. However, when facing some fragile industrial products, robots with rigid gripper will cause damage to them. The soft gripper was then proposed due to its flexible movement ability and adaptability to the environment. The soft gripper has broad application prospects in the fields of medicine, archaeology and bionics. It has attracted extensive attention from experts and scholars across the world [4-6].

Suzumori Yasuichi of Jinggangshan University in Japan developed a soft four-finger robot [7]. The manipulator is composed of four fingers, made of silicone material, and driven by compressed air. George M. Whitesides [8] of Harvard University and others developed a new type of soft manipulator with a 6-finger structure, as shown in Figure 1 [8]. The soft manipulator claw is made of silica gel and has 6 finger petals, which can be deformed by inflating air pressure into it, and can flexibly grasp objects of different shapes or fragile, with strong adaptability.

\footnotetext{
${ }^{1}$ Corresponding Author.
} 

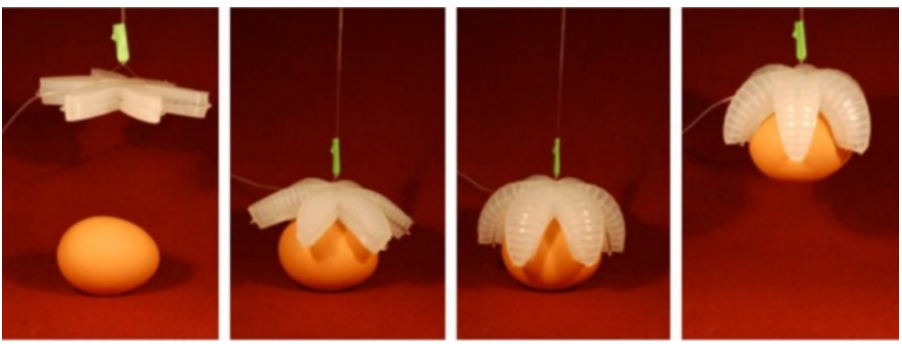

Figure 1. Six-finger silicone soft manipulator

Wei et al. [9] designed a pneumatic soft manipulator based on fiber-reinforced actuators. The manipulator was composed of three fiber-reinforced soft fingers, and the soft fingers are made from silicone gel by casting process. The gripping claw is stable and easy to control. In 2016, the team of Professor Wen Li of Beihang University ${ }^{[10]}$ developed a soft gripper that can change the effective gripping length, as shown in Figure 2 [10]. The soft gripper consists of four independent fingers, which had evenly distributed small air chambers. It concluded that the objects with different sizes prefer a certain effective finger length for generating maximum pull-off force.
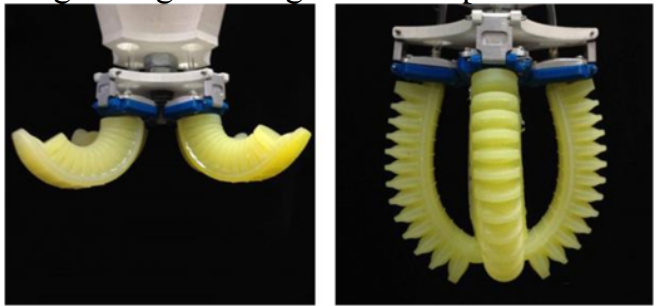

Figure 2. Soft gripper with variable effective grabbing length

In order to increase the rigidity of the soft finger, this paper designs a pneumatic soft finger with endoskeleton to enhance the stiffness and gripping stability of the finger. This will enable wider application of soft grippers. As for the human hand, there are bones inside the finger, allowing the finger gripping objects in different modes in the 3D space.

\section{Design and fabrication}

\subsection{Structural design}

The existence of embedded bones can play a role in limiting the elongation of the pneumatic soft finger. Therefore, the multi-cavity fast-type pneumatic soft finger with the least air consumption was used as the driving layer of the finger with endoskeleton. As shown in Figure 3, it was a multi-cavity fast pneumatic soft finger. The whole finger had 3 sections, each of which was composed of 4 air chambers with the same size.

\subsection{Production of the soft finger}

The mold and endoskeleton used polylactic acid material (PLA). The mechanical properties of PLA materials [11], and printing parameters are shown in Table 1. In the initial study, 5 kinds of silicone materials were chosen for the pneumatic soft finger, namely T00, T10, T20, T30, E630. Through comprehensive judgments on the fabricated 
finger, the most suitable silicone material E630 was selected as the material for making the mainbody of the pneumatic soft finger.

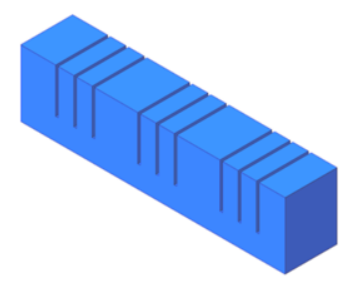

(a). Three-dimensional view

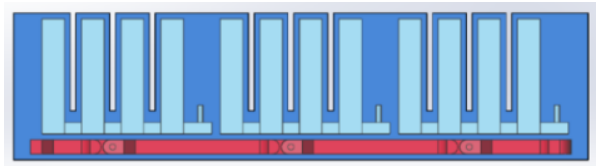

(b).Front view

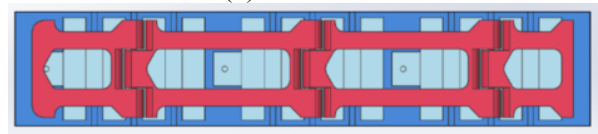

(c). Bottom view

Figure 3. Pneumatic soft finger

Table 1. PLA material properties

\begin{tabular}{ccccc}
\hline Material & $\begin{array}{c}\text { Printing } \\
\text { temperature }\left({ }^{\circ} \mathbf{C}\right)\end{array}$ & $\begin{array}{c}\text { Tensile } \\
\text { Strength (MPa) }\end{array}$ & $\begin{array}{c}\text { Elastic } \\
\text { Modulus (MPa) }\end{array}$ & $\begin{array}{c}\text { Poisson's } \\
\text { ratio }\end{array}$ \\
\hline CR-PLA & $195-210$ & 60 & 3000 & 0.25 \\
\hline
\end{tabular}

Taking into account the complexity of the internal cavity of the finger with endoskeleton, it was divided into two parts for casting, the upper layer and the bottom layer. The upper molds were used for casting the inner and outer shapes of the gas chamber respectively, and the two molds were connected by screws. All molds were printed by a 3D printer, and the printed molds are shown in Figure 4 . The basic 3D printing setting was $0.2 \mathrm{~mm}$ for the layer height, $0.8 \mathrm{~mm}$ for the wall thickness, and $50 \%$ for the filling density. For the embedded bone, shown in Figure 5, the printing filling density was $100 \%$.

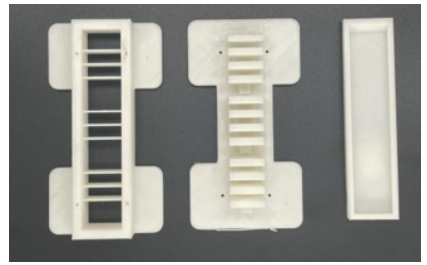

Figure 4. Physical image of the mold

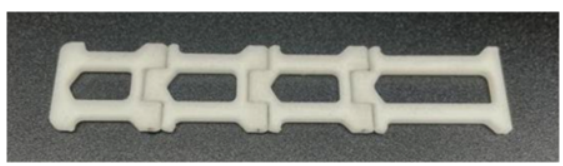

Figure 5. Endoskeleton structure

The production of the pneumatic soft finger with endoskeleton requires printed molds, embedded bone, sandpaper, paper clips, E630 silica gel solution, measuring cup, syringe, stirring rod and other materials. The produced pneumatic soft finger with endoskeleton is as presented in Figure 6.

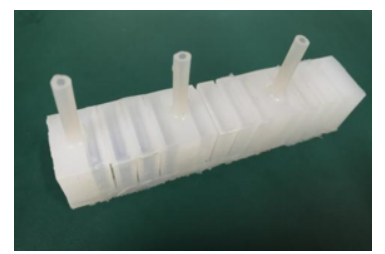

Figure 6. The final finger product 


\section{Finite element analysis}

\subsection{Material model}

The material used in the soft finger studied in this article was silica gel, which was a typical super-elastic material. The constitutive model of silica gel can be expressed by strain energy density. Common constitutive models include Ogden model, Yeoh model, etc. Generally speaking, the Yeoh model has good adaptability within $100 \%$ deformation and is the preferred constitutive model when analyzing the deformation of silica gel ${ }^{[12]}$.

The strain energy potential energy $\mathrm{W}$ is regarded as the strain energy density function of the deformation tensor invariant or the function expressed by the principal elongation ratio ${ }^{[13]}$.

$$
W=W\left(I_{1}, I_{2}, I_{3}\right)=W\left(\lambda_{1}, \lambda_{2}, \lambda_{3}\right)
$$

where I1, I2, I3 are deformation tensor invariants, and $\lambda 1, \lambda 2, \lambda 3$ are the main elongation ratios, all of which are dimensionless.

In the calculation, the energy density function in the form of binomial parameters is generally used. The typical binary parameter form expression of Yeoh model is as follows:

$$
W=C_{10}\left(I_{1}-3\right)+C_{20}\left(I_{1}-3\right)^{2}
$$

where $\mathrm{Cij}$ is a dimensionless relational constant.

The tensile sample is shown in Figure 7. The silica material sample was dumbbellshaped, with a total length of $115 \mathrm{~mm}$, a thickness of $2.5 \mathrm{~mm}$, a stretched part length of $33 \mathrm{~mm}$, a width of $6 \mathrm{~mm}$, and the radius of the transition part is $14 \mathrm{~mm}$ and $25 \mathrm{~mm}$, respectively. The sample mold was printed by a $3 \mathrm{D}$ printer. A total of three samples of the same material, as shown in Figure 8, and finally the data in the middle was selected as the tensile data of silica gel ${ }^{[14]}$.

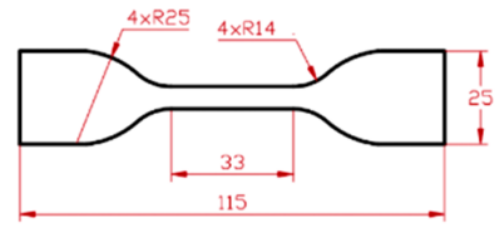

Figure 7. Tensile sample (unit: $\mathrm{mm}$ )

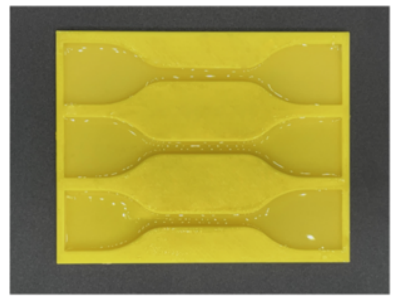

Figure 8. The actual tensile sample

The rubber tensile test instrument is QLW-5E microcomputer-based electronic tensile testing machine, and the experiment setup of uniaxial tensile test is shown in Figure 9. Repeat the tensile test on a single material three times, and select the group with the largest deformation under the same tensile force as the result of the tensile test. By processing the obtained load and deformation displacement data sets, the data set of engineering stress and engineering strain can be obtained, as shown in Figure 10. 

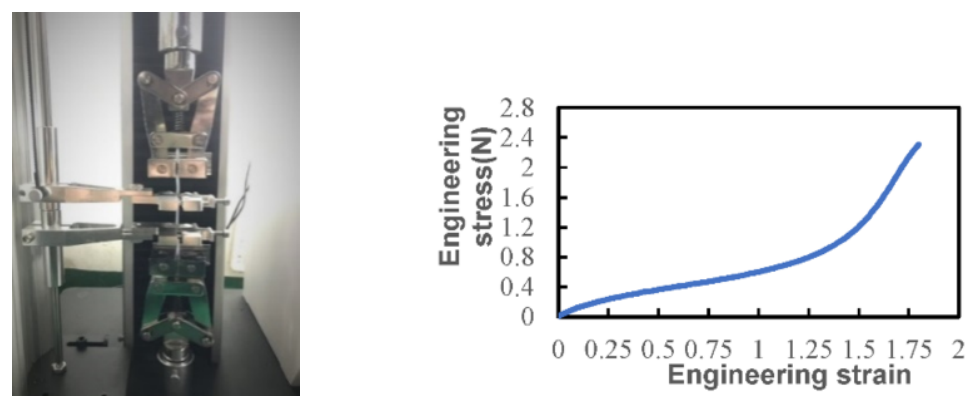

Figure 9. Uniaxial tensile test diagram of silica gel Figure 10. Relationship between engineering stress and engineering strain

The CR-PLA model 3D printing material, with a Young's modulus of $3000 \mathrm{MPa}$ and a Poisson's ratio of $0.25^{[15]}$. The silicone material is E630, and the constitutive model is Yeoh.

\subsection{Finite element modeling}

In this study, a single finger was fixed at one end, so the simulation uses the same fixing method as the real object. The loading and boundary condition are shown in Figure 11. The uniform pressure load was applied to the internal surface of the air chambers. The whole finger is meshed to get the embedded bone finger mesh as shown in Figure 12. The mesh size is 3, and the element type of E630 material and PLA material was $\mathrm{C} 3 \mathrm{D} 10 \mathrm{H}$.

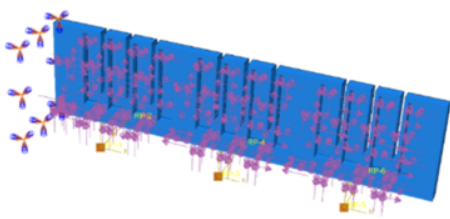

Figure 11. Loading and boundary conditions

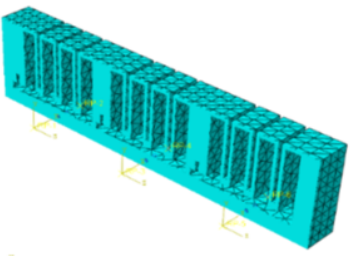

Figure 12. Pneumatic soft finger mesh with endoskeleton

\section{Bending performance analysis}

The pneumatic soft finger with endoskeleton was designed with three air chambers, i.e. two joints, which can control the bending separately. The bending degree of the pneumatic soft finger with all three air chambers pressurized is shown in Figure 13. As the air pressure increases, the stress was mainly concentrated in the connections of the air cavities. The deformation during pressurization was demonstrated by three deformed shape under $10 \mathrm{kPa}, 30 \mathrm{kPa}$ and $50 \mathrm{kPa}$ pressures, respectively, as shown in Figure 14. As the air pressure increases, the bending of the fingertips increases. 


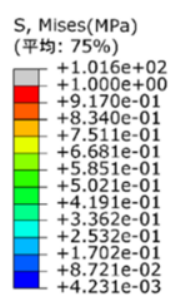

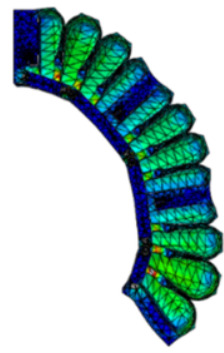

(a) Stress contour plot
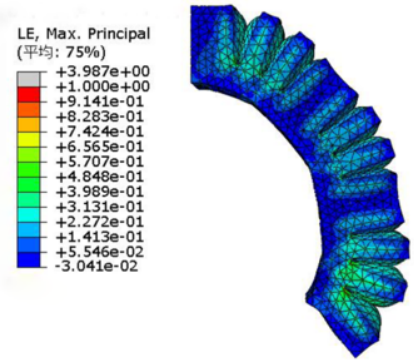

(b) Logarithmic strain cloudchart

Figure 13. Bending results under internal pressure

According to the Abaqus simulation, the relationship between the air pressure and the bending angle under different air pressures was obtained. The experiment was also carried out to verify the numerical model. The comparison of bending performance was shown in Figure 15. As the air pressure increases, the bending angle of the finger gradually increases linearly at low air pressure.

$10 \mathrm{kPa}$
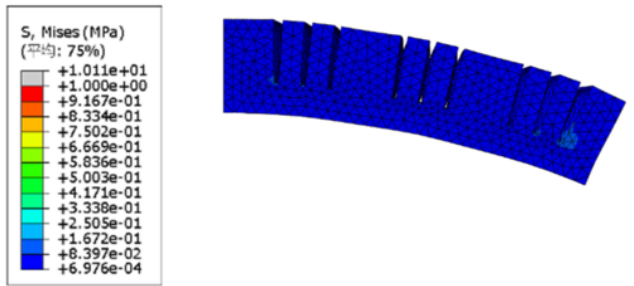

$30 \mathrm{kPa}$
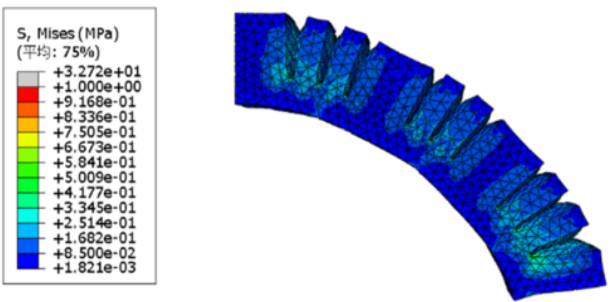

$50 \mathrm{kPa}$
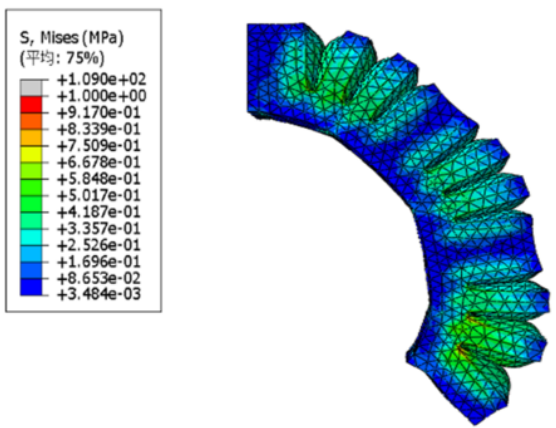

Figure 14. The simulation results of the pneumatic soft finger in different air pressures 


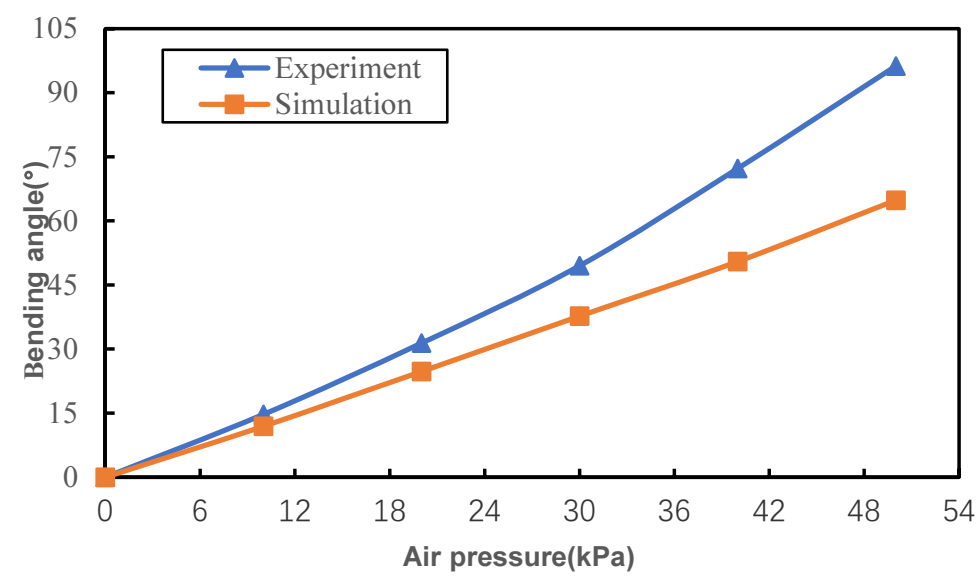

Figure 15. The relationship between the air pressure and the bending angle of the finger in all air chambers

\section{Conclusions}

This paper presented an innovative design of a pneumatic soft finger with endoskeleton. The main research conclusions are as follows:

(1) The proposed bionic human finger exhibited good bending performance when air pressure was applied, showing promising application. The embedded bone structure can increase the stiffness and withstand lateral bending moment. The finger had three sections, i.e., two joints, which can bend independently.

(2) The finger was fabricated using 3D printing technology and casting technology, the mold and endoskeleton was printed with polylactic acid material (PLA). The soft body was cast with silica gel materials. This fabrication method was proved reliably to produce complex soft fingers.

(3) Through comparison with experiment results, the finite element model can be used to simulate the bending deformation, providing an efficient tool to facilitate soft finger design.

\section{References}

[1] Tianmiao W. Thoughts on the Development of Industrial Robots. Robot Technology and Application, 2 (2004), 1-4.

[2] Zixing C. The Development Trend of Robot Technology in the 21st Century. Journal of Nanjing University of Chemical Technology (Natural Science Edition), 4 (2000), 73-78.

[3] Jiafan Z. Research on basic Theory and Application Technology of Human-machine Intelligence System Based on Flexible Exoskeleton. Zhejiang: Zhejiang University; 2009.

[4] Li J, Hegao C, Hong L. Journal of Mechanical Engineering, 4 (2004), 143.

[5] Yu X. Design and Experimental Research of Pneumatic Soft Manipulator. Southeast University. 2016.

[6] Tianmiao W, Yufe H, Xingbang Y, Li W. Journal of Mechanical Engineering, 53 (2017), 13, 1-13.

[7] Taogang H, SU Haohong. Science and Technology Review; 35 (2017), 18, 20-28.

[8] Ilievski Filip, Aaron MD, Robert SF. Soft robotics for chemists. Angewandte Chemie (International ed. in English), 50 (2011), 8, 1930-1935.

[9] Shujun W. Design and Control of Soft Manipulator Based on Pneumatic Actuator. Shanghai Jiaotong University; 2018. 
[10] Yufei H, Zheyuan G,Zhexin X. Universal soft robot Gripper with Variable effective Length .In: Control Theory Committee of The Chinese Society of Automation, Chinese Society of System Engineering. Control Theory Committee of The Chinese Association of Automation, Systems Engineering Society of China: Control Theory Committee of the Chinese Association of Automation; 6 (2016), 6109-6114.

[11] Zewen F,Xinyu Z, Shuai Q. Preparation and biocompatibility of poly (lactic acid)/polyethylene glycol/hydroxyapatite porous bone scaffolds by 3D printing .Journal of Materials Engineering; 2021, 49 (2021), 4, 135-141.

[12] Sheng Q, Yimin L, Xianqi Y. Rubber Science and Technology, 16 (2018), 5, 5-10.

[13] Jiazhao C, Minxiang H, Xueren W. Journal of Materials Review, 29 (2015), S1, 118-120.

[14] Han Z. Research on Grasping Performance of Pneumatic Soft Manipulator. Xi 'an University of Technology, 2019.

[15] Connolly F, Conor J.Bertold WK. Automatic design of fiber-reinforced soft actuators for trajectory matching. Proceedings of the National Academy of Sciences, 114 (2017), 1, 51-56. 